\title{
Longitudinal Reading Performance Comparison and its Variation with Print, Mobile and Social Media Tools
}

\author{
https://doi.org/10.3991/ijet.v16i13.21563 \\ Li Xiangming $(\bowtie)$ \\ Tsinghua University, Beijing, China \\ lixmesz.tsinghua.edu.cn \\ Xuening Li \\ Guangxi University for Nationalities, Nanning, China \\ Menghong Song \\ Jiangnan University, Wuxi, China \\ Jingshun Zhang \\ Florida Gulf Coast University, Florida, USA
}

\begin{abstract}
A plethora of previous literature generated the mixture of results by comparing the reading outcome between digital and printed contexts. Further, the cross-sectional study above failed to yield the findings about the multisession performance over a prolonged period of time as the reading activities proceeded. To address the gap, this study further explored the reading performance over a period of 12 weeks by designing the four-session frames for three different reading contexts of print, mobile and social media. Reading performance scores were gathered from 186 postgraduate students from a research university in P.R. China.

One-way ANOVA was conducted to compare the reading outcome among three reading contexts within each temporal session. The integrated statistical results showed that reading in social media produced the highest performance and reading in mobile the lowest performance across all the four temporal sessions. Longitudinal study revealed the consistency of reading performance in social media and in mobile with respectively high and low caliber over the four the reading sessions. Reading in print generated the performance in between except in the second temporal session. Future efforts could direct to the comparison of text length between short and long with different textual genres.
\end{abstract}

Keywords — Longitudinal; Mobile; Reading Performance; Social Media; Variation; Print

\section{$1 \quad$ Introduction and Literature Review}

Various reading modes have proliferated due to the newly emerged technological tools. Besides reading in traditional paper form, learners involved themselves in read- 
ing tasks facilitated by mobile learning apps, social media and other technological tools. A major line of research focused on the reading performance comparison in different reading situations ranging from traditional paper form to the digital tools [1, $2,3]$. Measuring reading performance was embraced in assessing the learner's reading efficacy along with attitudes or motivation $[4,5,6,7]$.

However, the prior studies yielded insufficient findings on two aspects. First, prior studies generated the mixed and conflicting conclusions about different reading modes, with better performance in paper materials [8], better performance in digital tools [9] and no significant difference between technology- and non-technologyintegrated situations [10]. Second, some scholars conducted the group comparison between the control group of traditional paper and experimental group or groups of technology, while others adopted the pre-and post-test methods to examine the effects of medium on reading performance based on individual difference $[11,12,13]$.

\section{Research Questions Proposed}

Therefore, more detailed information of the performance variation, especially of their group comparison over a period of time length were yet to explore in the aforementioned cross-sectional studies of group comparison and the longitudinal study of comparison between the beginning and the end.

In a 12-week long longitudinal study, this current study aimed to compare the reading performance in different reading contexts across the four temporal sessions. Altogether 186 participants were assigned the reading assignment and reading comprehension exercises after weekly class. Two research questions were proposed:

Q1: Is there any significant difference of reading performance comparison during each reading session?

Q2: Is there any shifting change in reading performance comparison across four reading sessions?

\section{$3 \quad$ Methodology}

\subsection{Context and participants}

Involved in the quasi-experiment were 186 participants for 12 consecutive weeks. Reading passages over 1000 English words along with reading comprehension tests were distributed to the participants as the after-class weekly assignments. Three modes of reading were designed, in which the first four-week session was for paper, the second four-week session for mobile phone of Rain Classroom and the third fourweek session for social media of WeChat group discussion.

The total number of 186 participants in this study were newly enrolled postgraduate students of various engineering disciplines from a research university in P.R. China with the average age of 22.4 . 


\subsection{Treatment of technological tools}

The social media of WeChat is the free-installed instant messenger which was developed and received wide popularity in China. Iqbal (2019) [14] recorded that the WeChat customers amounted to more than one billion per month. Participants utilized one of the functions called WeChat group discussion in this study.

Rain Classroom is a mobile learning tool newly developed by Tsinghua University in 2016. Participants performed its functions in mobile as Rain Classroom built in its public account in the WeChat $[15,16]$. In this current study, the teacher fed the reading materials and reading comprehension exercises. The participants as end users were expected to finish reading and answer the question. The answers and reading scores of participants were simultaneously fed to the teacher's end.

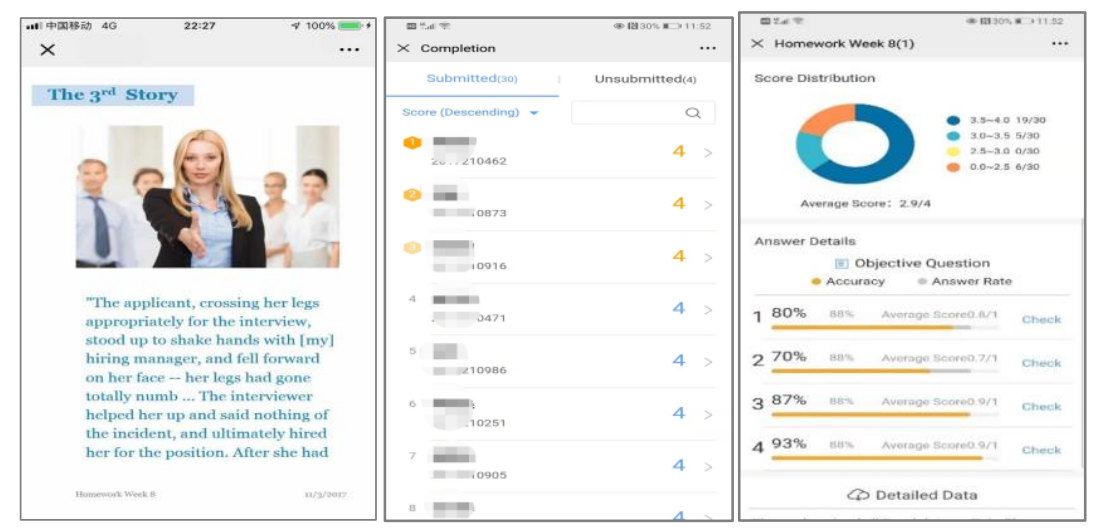

Fig. 1. Screen Shots of Reading Assignments and Test Scores from Teacher's End

\subsection{Instruments and data analysis}

Demographic Information Questionnaire: The demographic information questionnaire collected the participants' personal data including name, age, disciplines.

The Reading Test: The multiple-choice questions were designed and distributed to the participants in examining their reading comprehension performance. The raw scores for four time slots in each reading mode counted 23 points in total. This study adopted the min-max normalization transformed from raw scores. The transformed data ranged from 0 to 1 for accuracy and minimal variance.

One-way between-subjects ANOVA was conducted to compare the reading performance across different reading modes within each reading time slot for four times. The data was analyzed using SPSS20.0. 


\section{$4 \quad$ Results}

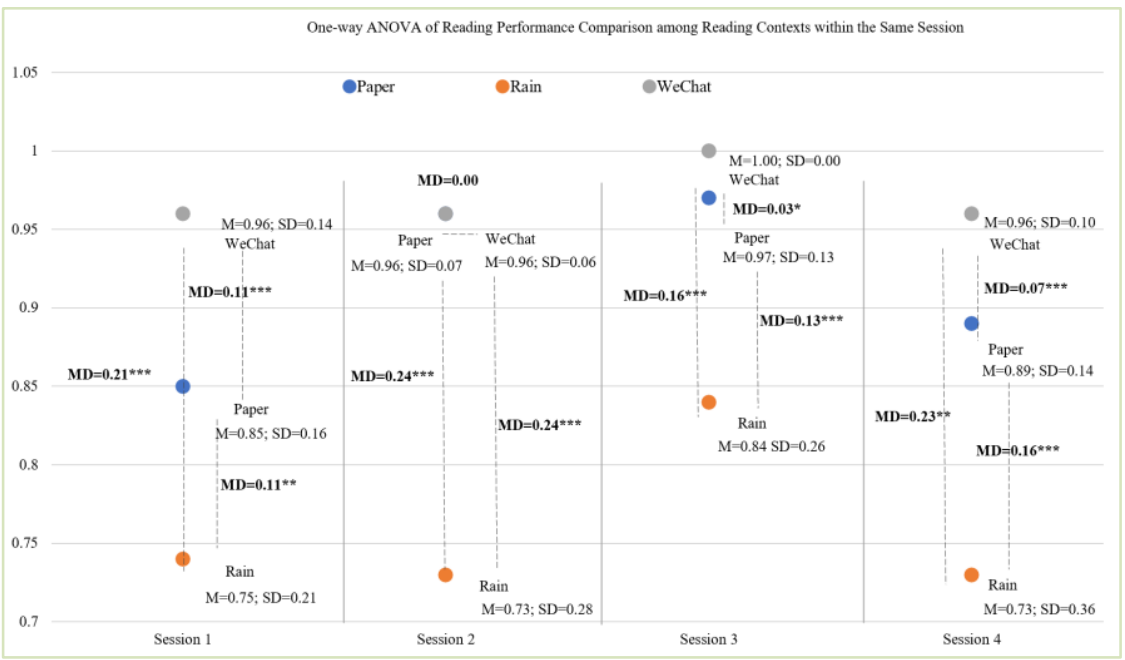

Fig. 2. One-way ANOVA of Test Scores across Four Sessions ( $\mathrm{N}=186)$

Note. $* \mathrm{p}<.05, * * \mathrm{p}<.01, * * * \mathrm{p}<.001 ; \mathrm{MD}=$ Mean Difference; WeChat=WeChat Group; Rain=Rain Classroom

As shown in Figure 2, One-way ANOVA was performed to examine the reading performance within the same sequential temporal session for reading activities in paper, mobile of Rain Classroom and social media of WeChat. Statistical results showed that there was a significant effect of group comparison in all the four temporal units $\quad(\mathrm{F} \quad(2,555)=68.68, \quad \mathrm{p}<0.001 ; \mathrm{F}(2,555)=117.18, \mathrm{p}<0.001 ; \mathrm{F}(2,555)=48.84$, $\mathrm{p}<0.001 ; \mathrm{F}(2,555)=47.42, \mathrm{p}<0.001)$

Post hoc comparisons using LSD showed that within the first temporal session, learners with WeChat group yielded the highest scores $(\mathrm{M}=0.96, \mathrm{SD}=0.14)$, followed by the scores by paper $(\mathrm{M}=0.85, \mathrm{SD}=0.16)$ and Rain Classroom $(\mathrm{M}=0.75, \mathrm{SD}=0.21)$, with $\mathrm{MD}=0.11, \mathrm{p}<0.001 ; \mathrm{MD}=0.11, \mathrm{p}<0.01 ; \mathrm{MD}=0.21, \mathrm{p}<0.001$ between WeChat group and the paper, between the paper and Rain Classroom, between WeChat group and paper respectively.

In the second temporal session, learners with paper and WeChat Group yielded the scores with no significant difference $(\mathrm{M}=0.965, \mathrm{SD}=0.06 ; \mathrm{M}=0.96 ; \mathrm{SD}=0.07)$. Yet both scores produced significantly higher scores than those using Rain Classroom $(\mathrm{M}=0.73, \mathrm{SD}=0.28)$, generating $\mathrm{MD}=0.00, \mathrm{p}>0.05 ; \mathrm{MD}=0.24, \mathrm{p}<0.001 ; \mathrm{MD}=0.24$, $\mathrm{p}<0.001$ between WeChat group and the paper, between the paper and Rain Classroom, between WeChat group and paper.

In the third temporal session, the WeChat Group again yielded the highest scores $(\mathrm{M}=1.00, \mathrm{SD}=0.00)$, followed by the scores of paper $(\mathrm{M}=0.97, \mathrm{SD}=0.13)$ and of Rain Classroom ( $\mathrm{M}=0.84, \mathrm{SD}=0.26$ ), producing $\mathrm{MD}=0.03, \mathrm{p}<0.05 ; \mathrm{MD}=0.13, \mathrm{p}<0.001$; $\mathrm{MD}=0.16, \mathrm{p}<0.001$ between WeChat group and the paper, between the paper and Rain Classroom, between WeChat group and paper. 
Also, in the fourth temporal session, the WeChat Group yielded the highest scores $(\mathrm{M}=0.96, \mathrm{SD}=0.10)$, followed by the scores by paper $(\mathrm{M}=0.89, \mathrm{SD}=0.14)$ and Rain Classroom $(\mathrm{M}=0.73, \mathrm{SD}=0.36)$ respectively, with $\mathrm{MD}=0.17, \mathrm{p}<0.001 ; \mathrm{MD}=0.16$, $\mathrm{p}<0.001 ; \mathrm{MD}=0.23, \mathrm{p}<0.01$ between $\mathrm{WeChat}$ group and the paper, between the paper and Rain Classroom, between WeChat group and paper respectively.

\section{Discussions}

Regarding the first research question, the current study produced the significant difference in group comparison across three different reading modes, which was applied to all the reading sessions. Specifically, WeChat group produced the highest reading scores and Rain Classroom the lowest performance throughout the four temporal units. Paper yielded the test scores in between all along the reading sessions except in the second temporal unit. Reading results of paper yielded no significant difference from WeChat group in the second time slot but significantly higher than mobile. This statistical result supported the findings in prior studies that reading in print generated better reading outcome than reading in mobile $[17,18]$.

As for the second research question, social media and mobile revealed the steady trend of performance in high and low caliber respectively over a stretch of prolonged reading sessions. This study further corroborated the previous literature in which the better reading outcome generated by paper over mobile in a cross-sectional study over a frozen moment.

\section{Conclusion}

This study attempted to unravel two confusing issues yet to address in the existing literature. One finding was about the superiority of social media in generating the reading outcome over the other traditional and mobile tools over a prolonged 4-week long period. The other finding referred to the sustained performance over four weeks of reading rather than the snapshots obtained from the cross-sectional study. Unlike the one-time measurement, more robust findings were yielded about the sustained performance and the variations in reading performance comparison among the four different temporal steps.

Still, there are some limits in this study while we deal with the findings with caution trying to generalize the findings to other settings. The reading materials were strictly refined to long text length and narratives. The participants were adult learners and there were no navigation links or hypertexts in the reading passages uploaded in mobile. Future efforts could direct to comparing the text length between short and long types with different textual genres. 


\section{$7 \quad$ Acknowledgment}

The present study was supported by Overseas Joint Research Grant, Shenzhen International Graduate School, Tsinghua University (HW2020003) and Guangdong Basic and Applied Basic Research Foundation (2021A1515012563).

\section{$8 \quad$ References}

[1] Delgado, P., Vargas, C., Ackerman, R., \& Salmerón, L. (2018). Don't throw away your printed books: A meta-analysis on the effects of reading media on reading comprehension. Educational Research Review. https://doi.org/10.1016/ j.edurev.2018.09.003.

[2] Lysenko, L. V., \& Abrami, P. C., (2014). Promoting reading comprehension with the use of technology. Computers \& Education, 75, 162-172. https://doi.org/10.1016/j.compedu. 2014.01.010

[3] Alamri, B. (2019). Reading Preferences of ESL Students: Electronic Texts vs. Printed. International Journal of Emerging Technologies in Learning, 14(4), 169-179. https://doi.org/ 10.3991/ijet.v14i04.9466

[4] Chen, C.-M., \& Lin, Y.-J. (2014). Effects of different text display types on reading comprehension, sustained attention and cognitive load in mobile reading contexts. Interactive Learning Environments, 24(3), 553-571. https://doi.org/10.1080/10494820.2014.891526

[5] Jang, B. G., Ryoo, J. H., \& Smith, K. C. (2020). Latent profiles of attitudes toward print and digital reading among adolescents. Reading and Writing, 1-25. https://doi.org/10.1007 $\underline{\text { /s11145-020-10104-7 }}$

[6] Liman Kaban, A., \& Karadeniz, S. (2021). Children's Reading Comprehension and Motivation on Screen Versus on Paper. SAGE Open, 11(1), 2158244020988849. https://doi.or g/10.1177/2158244020988849

[7] Abuloum, A., Farah, A., Kaskaloglu, E., \& Yaakub, A. (2019). College Students' Usage of and Preferences for Print and Electronic Textbooks. International Journal of Emerging Technologies in Learning, 14(7), 80-97. https://doi.org/10.3991/ijet.v14i07.9871

[8] Ackerman, R., \& Goldsmith, M. (2011). Metacognitive regulation of text learning: Onscreen versus on paper. Journal of Experimental Psychology: Applied, 17, 18-32. https://doi.org/10.1037/a0022086

[9] Cheung, A. C., \& Slavin, R. E. (2012). How features of educational technology applications affect student reading outcomes: A meta-analysis. Educational Research Review, 7(3), 198-215. https://doi.org/10.1016/j.edurev.2012.05.002

[10] Singer, L. M., \& Alexander, P. A. (2017b). Reading on paper and digitally: What the past decades of empirical research reveal. Review of Educational Research, 87, 1007-1041. https://doi.org/10.3102/0034654317722961

[11] Hou, J., Rashid, J., \& Lee, K. M. (2017). Cognitive map or medium materiality? Reading on paper and screen. Computers in Human Behavior, 67, 84-94. https://doi.org/10.1016 /j.chb.2016.10.014

[12] Jeong, Y.J. (2018). Comparison of Cognitive Workload Differences Across Paper, Computer, and Tablet for Short Passage Reading Tasks (Doctoral dissertation). Retrieved from http://s-space.snu.ac.kr/bitstream/10371/143716/1/000000153116.pdf .

[13] Lauterman, T., \& Ackerman, R. (2014). Overcoming screen inferiority in learning and calibration. Computers in Human Behavior, 35, 455-463. https://doi.org/10.1016/j.chb.2014 $\underline{.02 .046}$ 
[14] Iqbal, Mansoor. (2019). WeChat Revenue and Usage Statistics (2019). Retrieved July 9th, 2019, from https://www.businessofapps.com/data/wechat-statistics/

[15] Xiangming, L., \& Song, S. (2018). Mobile technology affordance and its social implications: A case of "Rain Classroom". British Journal of Educational Technology, 49(2), 276291. https://doi.org/10.1111/bjet.12586

[16] Xiangming, L., Liu, M., \& Zhang, C. (2020). Technological impact on language anxiety dynamic. Computers \& Education, 150, 103839. https://doi.org/10.1016/j.compedu.2020. $\underline{103839}$

[17] Persson, V., \& Nouri, J. (2018). A systematic review of second language learning with mobile technologies. International Journal of Emerging Technologies in Learning, 13(2), 188-210. https://doi.org/10.3991/ijet.v13i02.8094

[18] Mangen, A., Walgermo, B. R., \& Brønnick, K. (2013). Reading linear texts on paper versus computer screen: Effects on reading comprehension. International Journal of Educational Research, 58, 61-68. https://doi.org/10.1016/j.ijer.2012.12.002

\section{Authors}

Li Xiangming is an Associate Professor in Shenzhen International Graduate School, Tsinghua University, P. R. China. Her research interests include mobile learning and instructional design in higher educational settings, and EFL teaching. Her Email: lixm@sz.tsinghua.edu.cn .

Xuening Li is a Full Professor at School of Foreign Studies, Guangxi University for Nationalities, P. R. China. His research interests mainly include computational linguistics and translation studies. His email: mr.lixn@qq.com .

Menghong Song is a Lecturer at School of Foreign Studies, Jiangnan University, P. R. China. Her research area mainly covers the ESL teaching and learning. Her email: songmenghong@qq.com .

Jingshun Zhang is an Associate Professor in College of Education, Florida Gulf Coast University, USA. His research interests mainly include classroom assessment, research methodology and cognitive diagnostic assessment. His email: jzhang@fgcu.edu .

Article submitted 2021-01-28. Resubmitted 2021-03-23. Final acceptance 2021-03-26. Final version published as submitted by the authors. 